\title{
Gravity reference at the Argentinean-German Geodetic Observatory (AGGO) by co-location of superconducting and absolute gravity measurements
}

\author{
Ezequiel D. Antokoletz ${ }^{1,4}$ (I) Hartmut Wziontek ${ }^{2} \cdot$ Claudia N. Tocho $^{1} \cdot$ Reinhard Falk $^{3}$
}

Received: 18 December 2019 / Accepted: 17 July 2020 / Published online: 8 August 2020

(c) The Author(s) 2020, corrected publication 2020

\begin{abstract}
The Argentinean-German Geodetic Observatory (AGGO) is a fundamental geodetic observatory located close to the city of La Plata, Argentina. Two high-precision gravity meters are installed at AGGO: the superconducting gravimeter SG038, which is in operation since December 2015, and the absolute gravimeter FG5-227, which has provided absolute gravity measurements since January 2018. By co-location of gravity observations from both meters between January 2018 and March 2019, calibration factor and instrumental drift of the SG038 were determined. The calibration factor of the SG038 was estimated by different strategies: from tidal models, dedicated absolute gravity measurements over several days and a joint approach (including the determination of the instrumental drift) using all available absolute gravity data. The final calibration factor differs from the determination at the previous station, the transportable integrated geodetic observatory, in Concepcion, Chile, by only $0.7 \%$, which does not imply a significant change. From the combined approach also the mean absolute level of the SG was determined, allowing to predict absolute gravity values from the SG at any time based on a repeatability of $12 \mathrm{~nm} / \mathrm{s}^{2}$ for the FG5-227 at AGGO. Such a continuous gravity reference function provides the basis for a comparison site for absolute gravimeters in the frame of the international gravity reference frame for South America and the Caribbean. However, it requires the assessment of the total error budget of the FG5-227, including the link to the international comparisons, which will be subject of future efforts.
\end{abstract}

Keywords AGGO - Superconducting gravimeter - Calibration factor - Instrumental drift · Absolute gravimeter . International Gravity Reference Frame · Gravity reference function

\section{Introduction}

The Argentinean-German Geodetic Observatory (AGGO) is a fundamental geodetic observatory located close to the city of La Plata, Argentina, where all major geodetic tech-

Ezequiel D. Antokoletz

eantokoletz@fcaglp.unlp.edu.ar

1 Facultad de Ciencias Astronómicas y Geofísicas, Universidad Nacional de La Plata, Paseo del Bosque s/n, La Plata, Argentina

2 Gravity Metrology, Federal Agency for Cartography and Geodesy (BKG), Karl-Rothe-Straße 10, Leipzig, Germany

3 Gravity Metrology, Federal Agency for Cartography and Geodesy (BKG), Richard-Strauss-Allee 11, Frankfurt am Main, Germany

4 National Scientific and Technical Research Council (CONICET), Buenos Aires, Argentina niques are co-located (Antokoletz et al. 2017; Wziontek et al. 2017). The superconducting gravimeter SG038 (SG) was put into operation at AGGO in December 2015, after it was moved from the previous station, the Transportable Integrated Geodetic Observatory (TIGO), located in Concepcion, Chile. Since then, it has been continuously recording gravity variations over time, providing the basis for a gravity reference function.

Calibration factor and instrumental drift of the SG038 were previously determined at station TIGO. The calibration factor was found to be $-736.5 \mathrm{~nm} / \mathrm{s}^{2} / \mathrm{V}$ with an estimated uncertainty of $0.8 \mathrm{~nm} / \mathrm{s}^{2} / \mathrm{V}$ from 3 years of combination with absolute gravity observations. The SG instrumental drift was estimated with $51.9 \mathrm{~nm} / \mathrm{s}^{2} /$ year, with an estimated uncertainty of $0.3 \mathrm{~nm} / \mathrm{s}^{2} /$ year, by combining more than $130 \mathrm{AG}$ observation epochs (Wziontek et al. 2016).

The SG038 was transported over $3000 \mathrm{~km}$ by truck from Concepcion, Chile, to La Plata, Argentina. Although Meur- 
ers (2012) found no significant changes in the calibration factor after moving an SG over $60 \mathrm{~km}$ without lowering the sphere, the SG038 suffered a much longer trip. Therefore, the calibration factor had to be checked after the installation at AGGO. In addition, the instrumental drift had to be determined after the new setup since a change in its behavior is normally expected after changes of the magnetic field when re-centering the sphere.

Since the SG was installed at AGGO, several studies were carried out. Antokoletz et al. (2017) have determined the time delay due to electronic components of the SG038 and obtained a first tidal model for AGGO, by analyzing the first 6 months of superconducting gravity records. Oreiro et al. (2018) have investigated the presence of non-tidal loading effects from the estuary of the Río de La Plata in the SG gravity time series. Mikolaj et al. (2019) have developed a local hydrology gravity model based on records of hydrological and meteorological signals and evaluated it against the gravity residuals of the SG038.

The time series of the SG038 used in the cited studies were pre-processed using the calibration factor previously determined at station TIGO. Moreover, Mikolaj et al. (2019) applies a preliminary instrumental drift to account for longterm changes. For a meaningful interpretation of the residual gravity signal and the use as a comparison reference, the calibration factor and instrumental drift of the SG038 had to be revised for possible changes caused by the transportation and re-centering of the sphere.

Preliminary information about the SG calibration factor can be obtained by combination with a theoretical Earth tide model, including ocean tide loading, atmospheric and polar motion effects on gravity. Although such an approach seems controversial since it does not improve tidal modeling, today's ocean models allow for a practical estimate. Independent estimates are possible from co-located absolute gravity measurements. In January 2018, the absolute gravimeter (AG) FG5-227 was installed at AGGO, enabling a precise determination of the instrumental properties (calibration factor and instrumental drift) of the SG038. The instrumental drift was computed from all AG measurements between January 2018 and March 2019. After the initial estimate from theoretical tides, the SG calibration factor was determined from extensive AG measurements of several days duration. The results are compared with the calibration factor determination at TIGO.

The establishment of the International Gravity Reference Frame (IGRF), as recommended by the Resolution No. 2 of 2015 and No. 4 of 2019 of the International Association of Geodesy (IAG), requires a continuous gravity reference function for reference and comparison sites (Drewes and Kuglitsch 2019). IGRF core stations provide a link to the International Terrestrial Reference Frame (ITRF), where core sites of the Global Geodetic Observing System (GGOS) play an essential role. Furthermore, the stations of the IGRF should also be linked to the International Height Reference Frame (IHRF). AGGO as a member of GGOS space geodesy network, fulfills all requirements to become the only station with these characteristics in South America and the Caribbean. The presented combination of AG and SG observations sets the basis to establish such a continuous gravity time series, which will serve as a reference for comparisons of absolute gravity meter on a regional level.

\section{Observations}

\subsection{Absolute gravity observations}

Absolute gravity measurements have been repeatedly performed at AGGO since it was set up. The FG5-227 is installed on a pillar with a side length of $1.1 \mathrm{~m}$ which is founded $4 \mathrm{~m}$ deep at a distance of about $1.5 \mathrm{~m}$ from the pillar of the $\mathrm{SG}$ (Fig. 1a, b; Antokoletz et al. 2017). Two similar pillars allow for absolute gravity meter comparisons. All pillars are deepened into the soil (bedrock is not available in the area) and isolated from the floor of the gravity laboratory to reduce micro-seismic noise and avoid vibrations from the helium compressor of the SG.

Typically, the AG observations made at AGGO have a duration of about $24 \mathrm{~h}$. In order to derive the calibration factor of the SG, three dedicated campaigns were performed during periods of spring tides, to enhance the signal-to-noise ratio (Van Camp et al. 2016). These observations had a duration of 3 to 5 days.

An AG observation arises from thousands of free fall experiments (drops), which are averaged in sections of 100 drops (sets). A standard processing has been carried out with g-Software version 9 (Micro-g Lacoste Inc. 2012), applying a 3-sigma criterion of the standard deviation with respect to the set mean value to eliminate outliers.

Individual drop gravity values were used and timedependent corrections, which are usually applied during processing (Earth tides, ocean tide loading, atmospheric loading effects and pole tides), were restored to obtain the uncorrected gravity measurements. This is required to determine the scale (or calibration) factor and useful for drift determination, which is usually based on mean values over an AG observation epoch. By using the uncorrected drop data, the impact of small discrepancies between models used in AG and SG processing to correct temporal gravity changes can be avoided, and further allows a combined determination of both the scale factor and instrumental drift (Wziontek et al. 2009), an approach which is not frequently used. Figure 2a depicts the AG drop observations acquired in August 2018, after restoring time-dependent corrections. 
(a)

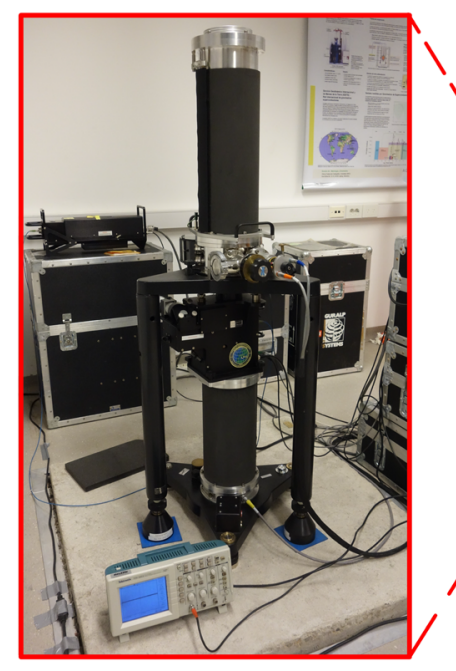

(b)

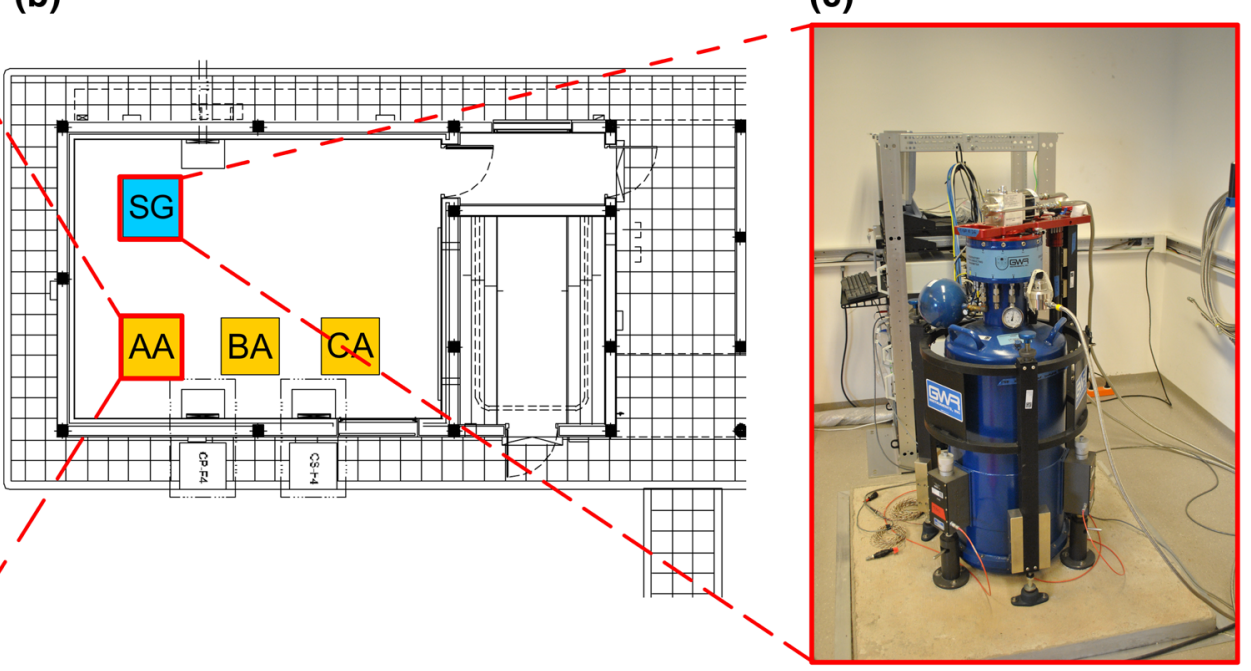

Fig. 1 Absolute gravimeter FG5-227 (a). Floor plan of the gravity laboratory (b). Superconducting gravimeter SG038 (c). BA and CA are the auxiliary pillars for absolute gravity comparisons

\subsection{Time series from the superconducting gravimeter}

The superconducting gravimeter SG038 (Fig. 1c) was the first remote-controlled instrument with a pre-set magnetic gradient for keeping the sphere levitated (Warburton et al. 2000). It is the only SG currently operated in South America and the Caribbean, and it contributes to the International Geodynamics and Earth Tides Service (IGETS, Voigt et al. 2016; Wziontek et al. 2017).

Continuous gravity time series are recorded with a sample rate of $1 \mathrm{~s}$. The raw data are then filtered and re-sampled to $10 \mathrm{~s}$ and $1 \mathrm{~min}$ with a finite impulse response (FIR) filter of 251 coefficients to suppress micro-seismic signals. The data filtered to $10 \mathrm{~s}$ were used and then interpolated to the respective observation epoch of each drop by cubic interpolation. Large disturbances caused by earthquakes were removed by introducing a gap. The time delay of $8.3 \mathrm{~s}$ caused by the electronic components was applied to the signal (Antokoletz et al. 2017). Figure $2 b$ illustrates the $S G$ gravity signal corresponding to the AG observation conducted in August 2018.

\section{Methodology}

\subsection{Instrumental drift}

Superconducting gravimeters are generally characterized by a very stable and low instrumental drift (Goodkind 1999; Hinderer et al. 2015), which can only be determined by combination of $\mathrm{AG}$ and $\mathrm{SG}$ observations. If both instruments

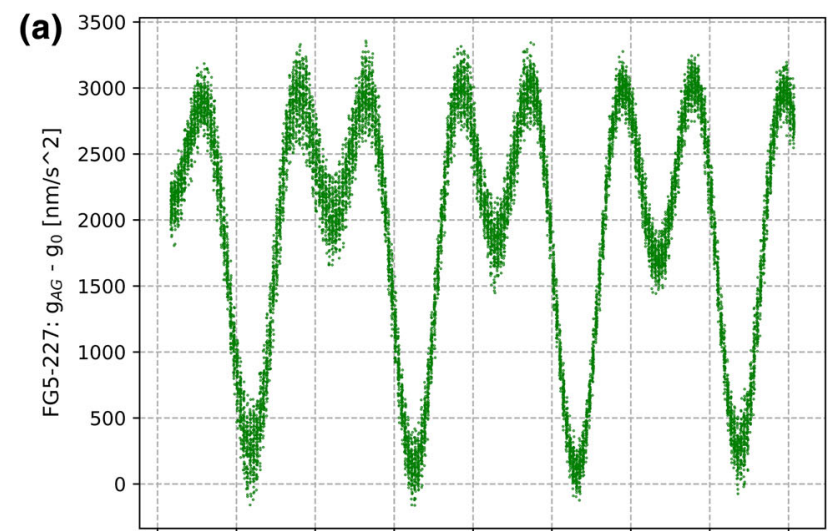

(b)

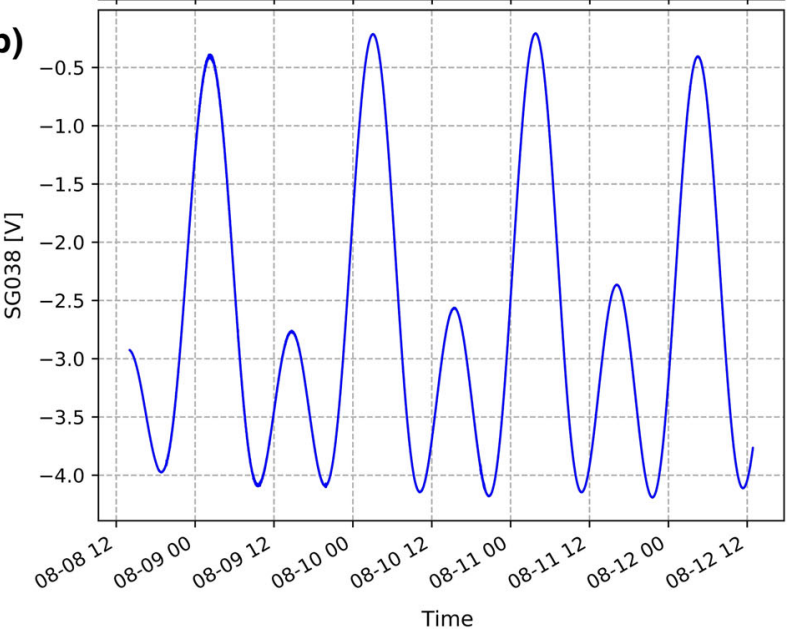

Fig. 2 AG observations made in August 2018, where outliers and a mean gravity value $\left(g_{0}\right)$ were removed $(\mathbf{a})$, and the SG measurements corresponding to the same period (b) 
are located at the same site, the instrumental drift of the SG can be separated from long-term geophysical trends in gravity, assuming that both instruments are affected by the same physical phenomena.

The determination of the instrumental drift was performed by a linear regression following equation (1):

$\mathrm{AG}_{\mathrm{obs}}+v=g_{0}+b_{1} \mathrm{SG}_{\mathrm{obs}}+c_{0}\left(t-t_{0}\right)$,

where $\mathrm{AG}_{\text {obs }}$ are the uncorrected drop $\mathrm{AG}$ values, $\mathrm{SG}_{\mathrm{obs}}$ are the corresponding SG observations, $v$ are the residuals, $b_{1}$ is the calibration or scale factor, $g_{0}$ is a constant representing the absolute reference value for the SG, $c_{0}$ is the SG drift, $t$ is the observation epoch, and $t_{0}$ is the reference epoch, corresponding to the first AG observation. The constant $g_{0}$ is essential to establish the gravity reference by the SG and allows to predict absolute gravity values.

Since the AG's noise level is typically 100 times higher than the SG's noise level (Van Camp et al. 2005), the SG measurements are assumed to be error free, and only errors in the absolute gravity measurements are taken into account (the residual term in Eq. 1). The given equation system is well determined and the adjustment can be performed by the least squares method. Individual AG drops of 15 observation epochs between January 2018 and March 2019 were used. Due to the amount of observations, not only the SG drift was adjusted but also the scale factor $\left(b_{1}\right.$ in Eq. 1$)$, in order to avoid fixing a variable.

\subsection{Calibration factor}

The calibration factor of SG038 at AGGO was evaluated by two strategies: (a) based on a theoretical Earth tide model, and (b) based on AG measurements from FG5-227. Although it is well known that the second method is in general more accurate, an estimation from theoretical Earth tides, including ocean tide loading, atmospheric effects and pole tides may help if no AG measurements are available (Neumeyer et al. 2002; Neumeyer 2010).

\subsubsection{Combination with theoretical Earth tide model}

The determination of the calibration factor was performed by a linear regression, following Eq. (2):

$g_{\text {tides }}+g_{\text {OTL }}+g_{\text {atm }}+g_{\text {pole }}+v=b_{0}+b_{1} \mathrm{SG}_{\text {obs }}$,

where $g_{\text {tides }}$ are the theoretical Earth tides, $g_{\text {OTL }}$ is the ocean tide loading effect, $g_{\text {atm }}$ is the atmospheric effect on gravity, $g_{\text {pole }}$ is the polar motion effect, $v$ are the residuals, $b_{0}$ a constant representing the mean value, $b_{1}$ the calibration factor and $\mathrm{SG}_{\mathrm{obs}}$ the $\mathrm{SG}$ observations in $\mathrm{V}$ units.
Theoretical Earth tides were obtained from the tidal potential catalogue of Hartmann and Wenzel (1995) together with synthetic Earth tide parameters (Dehant et al. 1999) to account for the elastic response of the Earth. The ocean tide loading effect was computed by using the FES2014b model (Carrere et al. 2016), with tidal parameters from the ocean tide loading provider of M.S. Bos and H.-G. Scherneck (http://holt.oso.chalmers.se/loading/). Atmospheric effects were computed based on a numerical weather model provided by the Atmospheric attraction computation service (Atmacs) of BKG (Klügel and Wziontek 2009) and the local air pressure record to improve the temporal resolution and the quality of high-frequency signal. Gravity variations due to polar motion were computed based on the EOP C04 series, published by the International Earth Rotation Service (IERS), using an amplitude factor of 1.16 (Wahr 1985).

The calibration factor was then adjusted by the least squares method, including SG data of an entire month (June 2016). The uncertainty estimates highly depend on the accuracy of the models involved in Eq. (2). In the case of the Hartmann-Wenzel's tidal catalogue, the accuracy was estimated with $0.01 \mathrm{~nm} / \mathrm{s}^{2}$ (Neumeyer 2010). For FES2014b, the uncertainty was estimated with $3 \mathrm{~nm} / \mathrm{s}^{2}$, from a comparison with FES2004 (Lyard et al. 2006) and EOT11a (Savcenko et al. 2012) ocean tide models. For Atmacs, the uncertainty was assumed to be better than $2 \mathrm{~nm} / \mathrm{s}^{2}$, based on Mikolaj et al. (2019) and polar motion effects on gravity were assumed to be error free, since polar coordinates can be accurately determined by space geodetic techniques (Hinderer et al. 2015).

\subsubsection{Combination with absolute gravity measurements}

The adjustment of the calibration factor from individual AG observation epochs with respective SG datasets was performed by a linear regression following Eq. (1). The drift term can usually be neglected for typical observation periods of a few days, assuming that the instrumental drift of the $\mathrm{SG}$ does not exceed a few tens of $\mathrm{nm} / \mathrm{s}^{2} /$ year (Hinderer et al. 2015), yielding to the classical relation (e.g., Hinderer et al. 1991; Sato et al. 1996; Francis et al. 1998; Falk et al. 2001; Tamura et al. 2004):

$\mathrm{AG}_{\mathrm{obs}}+v=d_{0}+b_{1} \mathrm{SG}_{\mathrm{obs}}$,

where now $d_{0}$ is a constant representing the mean AG value of the observation epoch.

AGs are usually operated assuring high instrumental stability. Sometimes, small changes in temperature or in the vertical alignment may cause slight trends. This is discussed in Meurers (2012) and it is solved by adding an additional time-dependent term at the right hand of Eq. (3). Then, the observation equation is similar to Eq. (1), where now $c_{0}$ is the trend parameter. Strictly speaking, $c_{0}$ is a combination of 


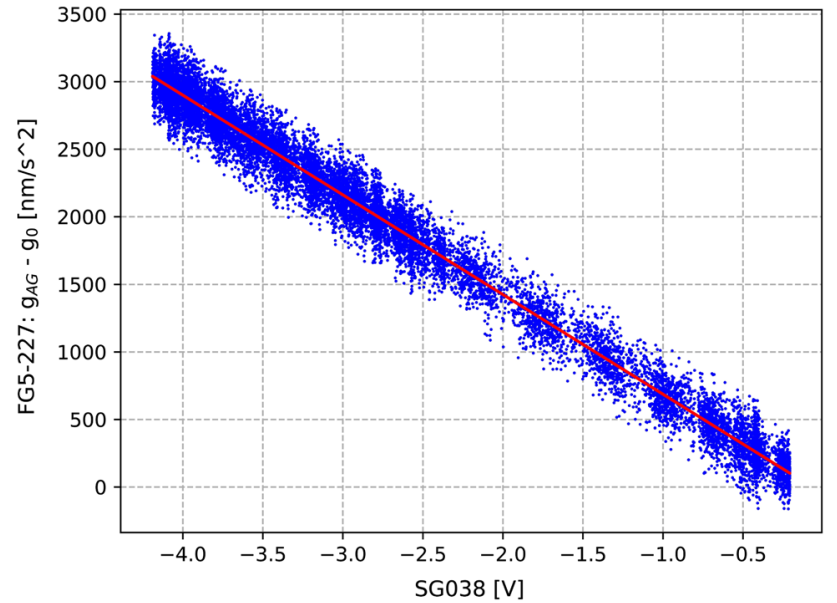

Fig. 3 Linear regression between AG and SG observations for the AG measurement made in August 2018, considering equation (3). $g_{0}$ is a mean gravity value

any trend in the AG measurement and the SG instrumental drift. As it was assumed that the SG drift is small enough to be neglected for a few days, $c_{0}$ can be attributed to possible trends in the absolute gravity observation.

Thus, the calibration factor was adjusted for each campaign with and without including the trend parameter in the linear regression. The uncertainty was estimated from the scatter of the individual AG drop measurements. Figure 3 illustrates the linear regression between AG and SG observations for the campaign made in August 2018, following Eq. (3).

\section{Results and discussion}

By including the drops of all AG observation epochs from January 2018 to March 2019, the SG038 instrumental drift was determined with $110 \mathrm{~nm} / \mathrm{s}^{2} /$ year from Eq. (1), with an estimated uncertainty of $4 \mathrm{~nm} / \mathrm{s}^{2} /$ year. The scale factor was simultaneously estimated in this combined approach. Figure 4 shows the SG residual curve for the same period before and after applying the drift correction, respectively, and the superimposed AG measurements. Earth tides were removed with a local model (Antokoletz et al. 2017), atmospheric effects were corrected based on Atmacs and the local air pressure record, as well as the effect of polar motion.

As mentioned in Sect. 1, the instrumental drift of SG038 at station TIGO/Concepcion was found to be $51.9 \mathrm{~nm} / \mathrm{s}^{2} /$ year, with an estimated uncertainty of $0.3 \mathrm{~nm} / \mathrm{s}^{2} /$ year. A change of this parameter was expected after re-centering the sensor at the new station. The estimated uncertainty is mainly limited by the amount of AG observation epochs. Once more AG observations are carried out at AGGO, the uncertainty of the SG instrumental drift will reduce.
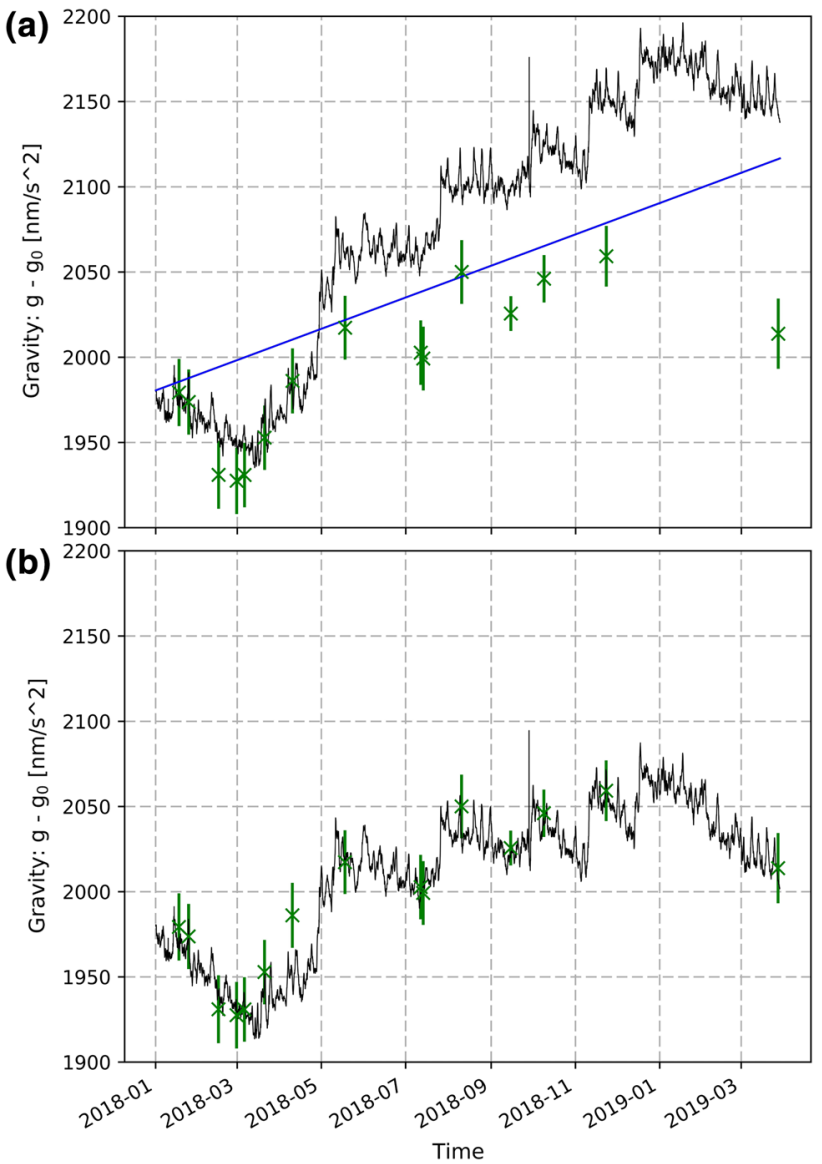

Fig. 4 SG residual curve from January 2018 to March 2019, where tides, atmosphere and polar motion are removed, before (a) and after (b) drift correction. Blue line: adjusted SG instrumental drift. Green markers: mean gravity values from AG observation epochs. The constant $g_{0}=9797327480 \mathrm{~nm} / \mathrm{s}^{2}$ is the absolute reference of the SG

Together with the calibration factor $b_{1}$ and the drift parameter $c_{0}$, the constant in Eq. (1) was determined to be $g_{0}=$ $9797327480 \mathrm{~nm} / \mathrm{s}^{2}$ with an uncertainty of $2.5 \mathrm{~nm} / \mathrm{s}^{2}$, with reference $t_{0}=2018-01-01$. This allows to transform the SG measurements into absolute gravity values, referred to the FG5-227 at pillar AA and the respective datum height of $125 \mathrm{~cm}$, assuming that gravity changes at the SG sensor position are equivalent to the AG within the limits of uncertainty. Figure 4 documents an excellent agreement between the SG curve and the AG measurements. The RMS of the differences between the AG and SG observations amounts to $12 \mathrm{~nm} / \mathrm{s}^{2}$, which reflects the accuracy of the FG5 and is a more realistic error estimate.

The absolute reference of the FG5-227 is controlled by measurements at Wettzell and Bad Homburg reference stations in Germany before it was shipped to Argentina. Therefore, AGGO can be linked to the CIPM comparison CCM.G-K2.2017 in Beijing, China, and the regional comparison EURAMET.M.G-K3 2018 in Wettzell, Germany. 
This set of three parameters provides a continuous absolute gravity reference function by the SG, presumed no instrumental steps occur. For SG038 the only step to be corrected is due to a planned interruption of the power supply on November 1st, 2016. With such a reference function, any other absolute gravimeter can be compared at AGGO, fulfilling the requirements of the IGRF.

The different calibration results are documented in Table 1. For comparison, the first row shows the scale factor determined at TIGO/Concepcion, Chile. The row highlighted in bold is based on theoretical models as described in Sect. 3.2. An exceptional low standard deviation of $0.002 \mathrm{~nm} / \mathrm{s}^{2} / \mathrm{V}$ was obtained, which reflects the high correlation between the SG observations and the used models during the period of 1 month. It demonstrates the importance of a long duration of parallel observations, as it is possible today with absolute quantum gravimeters. However, the low value does not characterize the uncertainty of the scale factor, as the uncertainties and systematic errors of the tidal models included in Eq. (2) remain unaccounted. The real uncertainty mainly depends on the quality of the ocean model and the computation of the respective loading effect and shows that even the theoretical approach has a potential nowadays, if no AG measurements are available. Besides this, the calibration factor confirms the previous determination at TIGO within the error bars and even agrees with the first dedicated AG campaign in May 2018.

The following rows in Table 1 (highlighted in italic) show the results for the calibration factor from dedicated absolute gravity measurements. Formally, the instrumental drift of the SG should be removed before estimating the scale factor. In practice, an instrumental drift of about $1 / 3 \mathrm{~nm} / \mathrm{s}^{2} /$ day is insignificant for a few days duration of each experiment. On the other hand, no significant trends were found which implies an excellent stability of the AG measurements. Only a slight trend of about $-7 \mathrm{~nm} / \mathrm{s}^{2} /$ day was found for the measurement made in July, which does not affect the scale factor significantly.
The last row (highlighted in bolditalic) shows the scale factor obtained from the combined adjustment of scale factor and drift from all drops (Eq. 1). Due to the larger number of observations, the uncertainty is lower and the so obtained calibration factor reflects well the average of the three campaigns. This consistency shows that no AG offsets nor steps in the $\mathrm{SG}$ record occurred.

Figure 5 depicts the histograms of the residuals of each adjustment of the selected periods, without including a trend parameter for the AG data. By a $\chi^{2}$-test (Lowry 2008), a normal distribution with a $99 \%$ of probability was found for all cases, meaning that there are no significant systematic effects omitted in the adjustment.

The estimated uncertainty of the calibration factor is, in all cases, below $0.2 \%$. However, from Table 1 the importance of specific AG campaigns for SG calibration factors determination during periods of spring tides becomes apparent. The calibration factor from the first AG campaign (May 2018) deviates about $2 \mathrm{~nm} / \mathrm{s}^{2} / \mathrm{V}$ from the other two determinations. This difference can be attributed to the difference in the peak-to-peak tidal amplitude reached during each experiment. Similar calibration factors were obtained from the other two AG campaigns (July and August 2018), where the tide variation is 200 to $300 \mathrm{~nm} / \mathrm{s}^{2}$ larger than in the first case. For this reason, the final value was obtained from an arithmetic weighted average of the three AG campaigns (rows highlighted in italic in Table 1), taking into account the peak-to-peak tidal amplitude of each experiment. It was found to be $-737.0 \mathrm{~nm} / \mathrm{s}^{2} / \mathrm{V}$, with an estimated uncertainty of $0.8 \mathrm{~nm} / \mathrm{s}^{2} / \mathrm{V}$, where the uncertainty was derived from Gaussian error propagation (Fornasini 2008). Final results with and without including a linear trend in the adjustment of the calibration factor are also shown in Table 1. Using the estimated precision for each experiment as weights results in the same values. The scale factor estimated together with the instrumental drift deviates by only $0.2 \%$, which is not significant.

Table 1 Scale factor (SF) and its standard deviation (SD) from different approaches, with and without including a linear trend in the adjustment. Final values from the weighted average (WA) are presented with and without including a linear trend in the adjustment. The previous determination at station TIGO is also shown

\begin{tabular}{|c|c|c|c|c|c|c|c|c|c|c|c|c|}
\hline Site & Date & $\begin{array}{l}\text { Period of } \\
\text { days }\end{array}$ & $\begin{array}{l}\# \text { of } \\
\text { values }\end{array}$ & $\begin{array}{l}\text { Tide } \\
\text { Variation } \\
{\left[\mathrm{nm} / \mathrm{s}^{2}\right]}\end{array}$ & $\begin{array}{l}\mathrm{SF} \\
\text { without } \\
\text { trend } \\
{\left[\mathrm{nm} / \mathrm{s}^{2} / \mathrm{V}\right]}\end{array}$ & $\begin{array}{l}\mathrm{SD} \\
{\left[\mathrm{nm} / \mathrm{s}^{2} / \mathrm{V}\right]}\end{array}$ & $\begin{array}{l}\mathrm{SF} \\
\text { with trend } \\
{\left[\mathrm{nm} / \mathrm{s}^{2} / \mathrm{V}\right]}\end{array}$ & $\begin{array}{l}\mathrm{SD} \\
{\left[\mathrm{nm} / \mathrm{s}^{2} / \mathrm{V}\right]}\end{array}$ & $\begin{array}{l}\text { WA } \\
\text { without } \\
\text { trend } \\
{\left[\mathrm{nm} / \mathrm{s}^{2} / \mathrm{V}\right]}\end{array}$ & $\begin{array}{l}\mathrm{SD} \\
{\left[\mathrm{nm} / \mathrm{s}^{2} / \mathrm{V}\right]}\end{array}$ & $\begin{array}{l}\text { WA } \\
\text { with trend } \\
{\left[\mathrm{nm} / \mathrm{s}^{2} / \mathrm{V}\right]}\end{array}$ & $\begin{array}{l}\mathrm{SD} \\
{\left[\mathrm{nm} / \mathrm{s}^{2} / \mathrm{V}\right]}\end{array}$ \\
\hline TIGO & 2009 to 2011 & - & 151,401 & - & -736.5 & 0.8 & - & - & - & - & - & - \\
\hline AGGO & June, 2016 & 30 & 256,660 & 2780 & -735.6 & 0.002 & - & - & - & - & - & - \\
\hline$A G G O$ & May 16, 2018 & 3 & 11,951 & 2740 & -735.6 & 1.5 & -735.6 & 1.5 & & & & \\
\hline$A G G O$ & July 12,2018 & 3 & 11,832 & 3030 & -737.9 & 1.5 & -737.3 & 1.4 & -737.0 & 0.8 & -736.8 & 0.8 \\
\hline$A G G O$ & August 8, 2018 & 5 & 14,990 & 2940 & -737.5 & 1.3 & -737.5 & 1.3 & & & & \\
\hline$A G G O$ & All drops & - & 63,266 & - & -737.2 & 0.7 & - & - & - & - & - & - \\
\hline
\end{tabular}

Bold: theoretical models. Italic: dedicated AG observation epochs. Bolditalic: from all AG measurements (drift computation) 
(a)

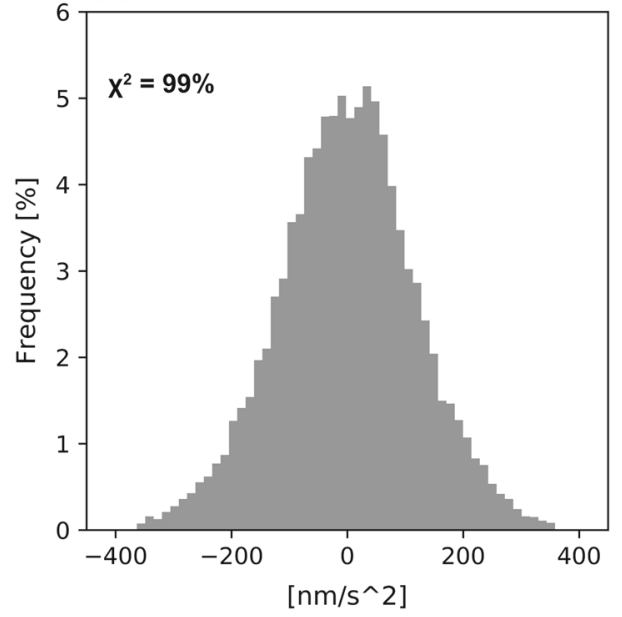

(b)

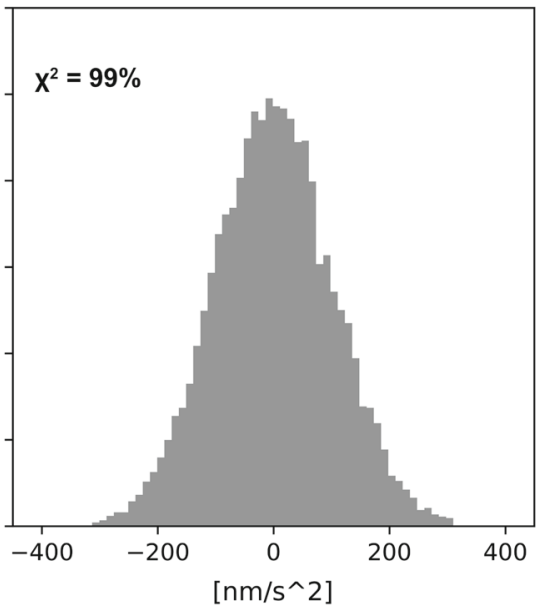

(c)

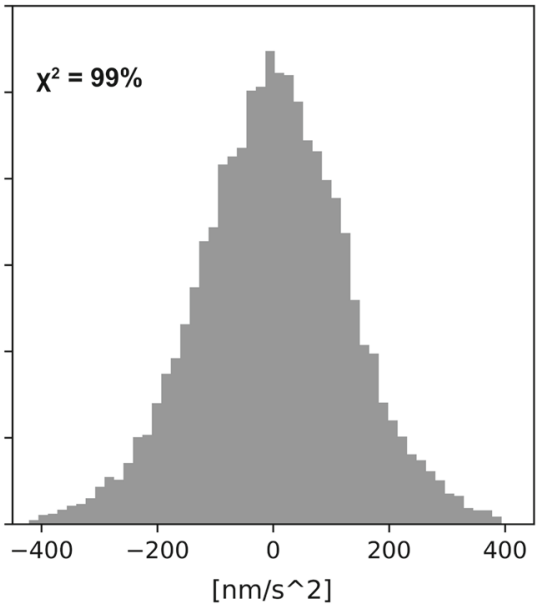

Fig. 5 Histogram of the residuals of each adjustment using AG data and Eq. (3). a May 16, 2018. b July 12, 2018. c August 8, 2018. The result of the $\chi^{2}$-test is shown for each adjustment

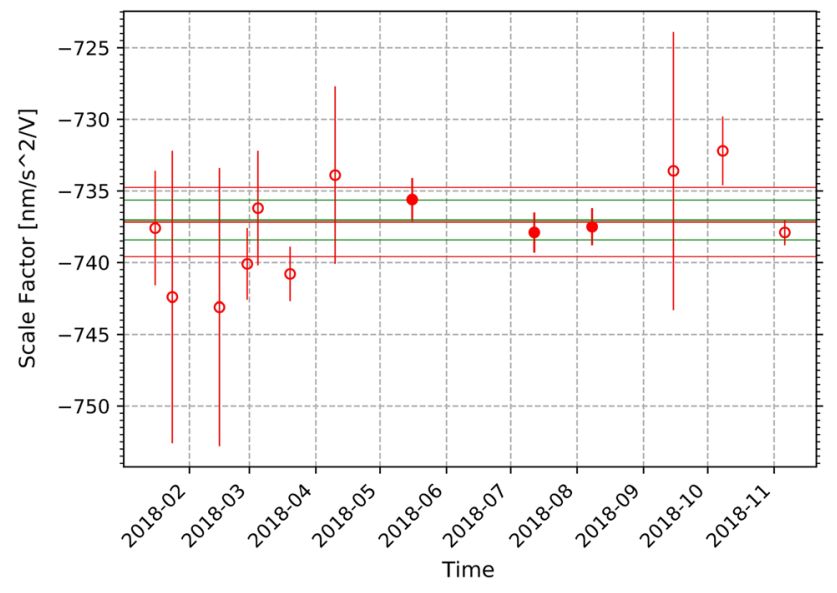

Fig. 6 Scale factor computed in each AG measurement with corresponding uncertainty bars. Filled red dots are the scale factor determinations for the specific campaigns in Table 1. Empty red dots are the scale factor determinations for all the AG observations. Red horizontal line: weighted mean of the results including all observations with uncertainty span. Green horizontal line: weighted mean of the results of the specific campaigns with uncertainty span

Apart from the specific calibration campaigns, the same procedure was applied to all observations made with the AG during this period, even if they were performed for regular absolute reference determination with an observation period of about 24 h. Figure 6 shows the results of each adjustment by using Eq. (3). A clear reduction of the scatter was achieved for the more extensive AG measurements, demonstrating the importance of longer observations over several days, as previously pointed out by e.g., Rosat et al. (2009), Hinderer et al. (1998) or Van Camp et al. (2016). Large uncertainties are also associated with lower peak-to-peak tidal amplitude during the experiment, causing a lower signal-to-noise ratio.
The current scale factor differs by only $0.7 \%$ from the previous determination at TIGO/Concepcion, Chile, which is not significant.

\section{Conclusions}

Some instrumental properties of the superconducting gravimeter SG038 installed at AGGO have been evaluated. Based on absolute gravity measurements with FG5-227 from January 2018 to March 2019, the calibration factor and the instrumental drift of SG038 were determined.

The SG instrumental drift was found to be $110 \mathrm{~nm} / \mathrm{s}^{2} /$ year from a simultaneous estimate of the calibration factor. Uncorrected gravity values of each individual drop were used to avoid the impact of models used to correct temporal gravity changes. The estimated uncertainty of the $\mathrm{SG}$ instrumental drift was found in $4 \mathrm{~nm} / \mathrm{s}^{2} /$ year. Together with the constant obtained from this approach the absolute gravity reference for the SG record was defined.

The calibration factor was computed from theoretical tides for an entire month, from three dedicated AG measurements and from the whole set of drops. The results of the three approaches agree within the error bars. The estimated uncertainty remained below $0.2 \%$ in all cases and the residuals of the adjustments show normal distributions, meaning that there are no systematic effects omitted.

The final scale factor was found from the three particular campaigns to be $-737.0 \mathrm{~nm} / \mathrm{s}^{2} / \mathrm{V}$ with an uncertainty of $0.8 \mathrm{~nm} / \mathrm{s}^{2} / \mathrm{V}$, as an arithmetic weighted average, taking into account the tidal amplitude of each campaign.

In contrast to Meurers (2012), trend estimates for individual AG measurements were not significant and have not affected the determination of the scale factor. This allows 
to conclude that no trends are needed for calibration factor determination if the stability of the AG is guaranteed. The SG calibration factor was also computed from all other AG measurements. These results show a considerable larger scatter, demonstrating the need to perform longer AG measurement periods. However, the overall scale factor estimate is in good agreement with the final value.

No significant changes from the previous determination of the scale factor at TIGO/Concepcion are recognized. The difference is below the expected accuracy of $0.1 \%$, following Baker and Bos (2003). In order to achieve a more accurate calibration factor (and possible changes on it), more precise methods to determine SG calibration factors should be considered, such as a calibration platform (Richter et al. 1995) or quantum sensors allowing for continuous operation over long periods (Freier et al. 2016).

With these results AGGO has been established as the only station providing a continuous gravity reference function in South America and the Caribbean, suitable for AG comparisons. The station is now well qualified to become one of the core stations of the International Gravity Reference Frame (IGRF), linked to the International Terrestrial Reference Frame (ITRF) and the International Height Reference Frame (IHRF; Tocho et al. 2020).

Acknowledgements This work was possible thanks to the AGGO's scientific directors, Dr. Claudio Brunini and Dr. Hayo Hase. The authors thank also I. Nowak, A. M. Cassino, M. Häfner, H. Hase, A. Lothhammer, A. Pasquaré and A. Reinhold for setup and operation of the absolute gravimeter and maintenance of the superconducting gravimeter. The authors sincerely thank the three anonymous reviewers for their comments and suggestions. They have contributed to considerably enrich this contribution.

Author contributions EA, HW and CT drafted and coordinated the work on the manuscript. EA, HW and CT processed the time series of the SG and computed the instrumental parameters of the SG. HW and $\mathrm{RF}$ processed the absolute gravity measurements.

Data availability statement The SG038 gravity time series are available at http://isdc.gfz-potsdam.de/. Absolute gravity results are available at http://agrav.bkg.bund.de/. The results of individual drop observations can be requested from BKG by contacting Dr.-Ing. Hartmut Wziontek. All data generated during the current study are available in this article.

Open Access This article is licensed under a Creative Commons Attribution 4.0 International License, which permits use, sharing, adaptation, distribution and reproduction in any medium or format, as long as you give appropriate credit to the original author(s) and the source, provide a link to the Creative Commons licence, and indicate if changes were made. The images or other third party material in this article are included in the article's Creative Commons licence, unless indicated otherwise in a credit line to the material. If material is not included in the article's Creative Commons licence and your intended use is not permitted by statutory regulation or exceeds the permitted use, you will need to obtain permission directly from the copyright holder. To view a copy of this licence, visit http://creativecomm ons.org/licenses/by/4.0/.

\section{References}

Antokoletz ED, Wziontek H, Tocho C (2017) First six months of superconducting gravimetry in Argentina. In: Vergos G, Pail R, Barzaghi $\mathrm{R}$ (eds) International symposium on gravity, geoid and height systems 2016 International Association of Geodesy Symposia, vol 148. Springer, Cham. https://doi.org/10.1007/1345_2017_13

Baker TF, Bos MS (2003) Validating earth and ocean tide models using tidal gravity measurements. Geophys J Int 152(2):468-485

Carrere L, Lyard F, Cancet M, Guillot A, \& Picot N (2016) FES 2014, a new tidal model-Validation results and perspectives for improvements. In: Proceedings of the ESA living planet symposium

Dehant V, Defraigne P, Wahr JM (1999) Tides for a convective earth. J Geophys Res Solid Earth 104(B1):1035-1058. https://doi.org/10. 1029/1998JB900051

Drewes H, Kuglitsch F (eds) (2019) IAG Reports 2015-2019 (Travaux de l'AIG vol 41), pp 147-149. https://iag.dgfi.tum.de/fileadmin/ IAG-docs/Travaux_2015-2019.pdf. Accessed 14 Feb 2020

Falk R, Harnisch M, Harnisch G, Nowak I, Richter B, Wolf P (2001) Calibration of the superconducting gravimeters SG1O3, C023, C0029 and CDO3O. J Geod Soc Jpn 47(1):22-27. https://doi.org/ 10.11366/sokuchi1954.47.22

Fornasini P (2008) The uncertainty in physical measurements: an introduction to data analysis in the physics laboratory. Springer, New York

Francis O, Niebauer TM, Sasagawa G, Klopping F, Gschwind J (1998) Calibration of a superconducting gravimeter by comparison with an absolute gravimeter FG5 in boulder. Geophys Res Lett 25(7):1075-1078. https://doi.org/10.1029/98GL00712

Freier C, Hauth M, Schkolnik V, Leykauf B, Schilling M, Wziontek H, Scherneck H-G, Müller J, Peters A (2016) Mobile quantum gravity sensor with unprecedented stability. In: Journal of physics: conference series, vol 723, no. 1. IOP Publishing, p 012050. https:// doi.org/10.1088/1742-6596/723/1/012050

Goodkind JM (1999) The superconducting gravimeter. Rev Sci Instrum 70(11):4131-4152

Hartmann T, Wenzel HG (1995) The HW95 tidal potential catalogue. Geophys Res Lett 22(24):3553-3556. https://doi.org/10. 1029/95GL03324

Hinderer J, Florsch N, Mäkinen J, Legros H, Faller JE (1991) On the calibration of a superconducting gravimeter using absolute gravity measurements. Geophys J Int 106(2):491-497. https://doi.org/10. 1111/j.1365-246X.1991.tb03907.x

Hinderer J, Amalvict M, Florsch N, Francis O, Mäkinen J (1998) On the calibration of superconducting gravimeters with the help of absolute gravity measurements. In: Proceedings of the thirteenth international symposium on earth tides, pp 557-564. https:// orbilu.uni.lu/bitstream/10993/3220/1/Hinderer\&al.-On\%20the \%20calibration\%20of\%20SG\%20-1998.pdf. Accessed 15 Dec 2019

Hinderer J, Crossley D, Warburton RJ (2015) Superconducting gravimetry, treatise on geophysics, 2nd edn, vol 3, pp 59-115. https://doi. org/10.1016/B978-0-444-53802-4.00062-2

Klügel T, Wziontek H (2009) Correcting gravimeters and tiltmeters for atmospheric mass attraction using operational weather models. J Geodyn 48(3-5):204-210. https://doi.org/10.1016/j.jog.2009.09. 010

Lowry R (2008) Concepts and applications of inferential statistics. Online Statistic Textbook. http://vassarstats.net/textbook/. Accessed 4 Aug 2020

Lyard F, Lefevre F, Letellier T, Francis O (2006) Modelling the global ocean tides: modern insights from FES2004. Ocean Dyn 56(56):394-415. https://doi.org/10.1007/s10236-006-0086-x 
Meurers B (2012) Superconducting gravimeter calibration by colocated gravity observations: results from GWR C025. Int J Geophys. https://doi.org/10.1155/2012/954271

Micro-g Lacoste Inc (2012) g9 user's manual, April 2012 version. Micro-g LaCoste, Lafayette

Mikolaj M, Reich M, Güntner A (2019) Resolving geophysical signals by terrestrial gravimetry: a time domain assessment of the correction-induced uncertainty. J Geophys Res Solid Earth 124:2153-2165. https://doi.org/10.1029/2018JB016682

Mikolaj M, Güntner A, Brunini C, Wziontek H, Gende M, Schröder S, Cassino AM, Pasquaré A, Reich M, Hartmann A, Oreiro FA, Pendiuk J, Guarracino L, Antokoletz ED (2019) Hydrometeorological and gravity signals at the Argentine-German geodetic observatory (AGGO) in La Plata. Earth Syst Sci Data 11:15011513. https://doi.org/10.5194/essd-11-1501-2019

Neumeyer J (2010) Superconducting Gravimetry. In: Xu G (ed) Sciences of geodesy - I. Springer, Berlin, Heidelberg. https://doi.org/ 10.1007/978-3-642-11741-1_10

Neumeyer J, Barthelmes F, Combrinck L, Dierks O, Fourie P (2002) Analysis results from the SG registration with the dual sphere superconducting gravimeter at SAGOS (South Africa). Bull Inf Marees Terr 135(10):607-10

Oreiro FA, Wziontek H, Fiore MME, D’Onofrio EE, Brunini C (2018) Non-tidal ocean loading correction for the Argentinean-German Geodetic Observatory using an empirical model of storm surge for the Río de la Plata. Pure Appl Geophys 175:1739-1753. https:// doi.org/10.1007/s00024-017-1651-6

Richter B, Wilmes H, Nowak I (1995) The Frankfurt calibration system for relative gravimeters. Metrologia 32(3):217. https://doi.org/10. 1088/0026-1394/32/3/010

Rosat S, Boy JP, Ferhat G, Hinderer J, Amalvict M, Gegout P, Luck B (2009) Analysis of a 10-year (1997-2007) record of time-varying gravity in Strasbourg using absolute and superconducting gravimeters: new results on the calibration and comparison with GPS height changes and hydrology. J Geodyn 48(3-5):360-365. https://doi. org/10.1016/j.jog.2009.09.026

Sato T, Tamura Y, Okubo S, Yoshida S (1996) Calibration of the scale factor of superconducting gravimeter at Esashi using an absolute gravimeter FG5. J Geod Soc Jpn 42(4):225-232

Savcenko R, Bosch W, Dettmering D, Seitz F (2012) EOT11a—global empirical ocean tide model from multi-mission satellite altimetry, with links to model results. https://doi.org/10.1594/PANGAEA. 834232 Supplement to: Savcenko R, Bosch W (2012): EOT11a Empirical Ocean Tide Model from Multi-Mission Satellite Altimetry. Deutsches Geodätisches Forschungsinstitut (DGFI), München, 89, 49 pp. https://doi.org/10013/epic.43894.d001
Tamura Y, Sato T, Fukuda Y, Higashi T (2004) Scale factor calibration of a superconducting gravimeter at Esashi Station, Japan, using absolute gravity measurements. J Geod 78(7-8):481-488. https:// doi.org/10.1007/s00190-004-0415-0

Tocho CN, Antokoletz ED, Piñón DA (2020) Towards the realization of the international height reference frame (IHRF) in Argentina. In: International association of geodesy symposia. Springer, Berlin. https://doi.org/10.1007/1345_2020_93

Van Camp M, Williams SDP, Francis O (2005) Uncertainty of absolute gravity measurements. J Geophys Res Solid Earth 110(B5):B05406. https://doi.org/10.1029/2004JB003497

Van Camp M, Meurers B, De Viron O, Forbriger T (2016) Optimized strategy for the calibration of superconducting gravimeters at the one per mille level. J Geod 90(1):91-99. https://doi.org/10.1007/ s00190-015-0856-7

Voigt C, Förste C, Wziontek H, Crossley D, Meurers B, Pálinkáš V, Hinderer J, Boy J-P, Barriot J-P, Sun H (2016) Report on the data base of the international geodynamics and earth tide service (IGETS), (Scientific Technical Report STR—Data ; 16/08). Potsdam: GFZ German Research Centre for Geosciences. https://doi. org/10.2312/GFZ.b103-16087

Wahr JM (1985) Deformation induced by polar motion. J Geophys Res Solid Earth 90(B11):9363-9368. https://doi.org/10.1029/ JB090iB11p09363

Warburton RJ, Brinton E, Reineman R, Richter B (2000) Remote operation of superconducting gravimeters. In: Proceedings of the workshop: high precision gravity measurements with applications to geodynamics and second GGP workshop, vol 17, pp 125-136

Wziontek H, Falk R, Hase H, Böer A, Güntner A, Wang R (2016) Continuous gravity monitoring in South America with superconducting and absolute gravimeters: more than 12 years time series at station TIGO/Concepcion (Chile). In: Geophysical research abstracts, vol 18, EGU2016-12195

Wziontek H, Falk R, Wilmes H, Wolf P (2009) Precise gravity time series and instrumental properties from combination of superconducting and absolute gravity measurements. In: Observing our changing earth. Springer, Berlin, pp 301-306. https://doi.org/10. 1007/978-3-540-85426-5_35

Wziontek H, Wolf P, Häfner M, Hase H, Nowak I, Rülke A Wilmes H, Brunini C (2017) Superconducting gravimeter data from AGGO/La Plata-level 1. GFZ data services. https://doi.org/10. 5880/igets.lp.11.001 